\title{
Comportamento ingestivo de cabras Alpinas em lactação alimentadas com dietas contendo diferentes níveis de fibra em detergente neutro proveniente da forragem 1
}

\section{Sérgio Carvalho², Marcelo Teixeira Rodrigues ${ }^{3}$, Renata Helena Branco ${ }^{4}$, Carla Aparecida Florentino Rodrigues 4}

\footnotetext{
1 Parte da tese de Doutorado em Zootecnia do primeiro autor

2 Grupo de Pesquisa em Agronegócios do Centro Universitário Feevale - Novo Hamburgo/RS.

${ }^{3}$ Departamento de Zootecnia da Universidade Federal de Viçosa/MG.

${ }^{4}$ Doutor em Zootecnia da Universidade Federal de Viçosa.
}

\begin{abstract}
RESUMO - Avaliou-se o efeito de diferentes níveis de fibra em detergente neutro proveniente da forragem (FDNF) na dieta sobre o comportamento ingestivo de cabras em lactação. Cinqüenta cabras da raça Alpina foram distribuídas em um delineamento inteiramente casualizado, com cinco tratamentos e dez repetições, sendo alimentadas à vontade com dietas contendo 20, 27, 34, 41 ou 48\% de FDNF. Foi utilizada uma ração completa com feno de tifton-85 (Cynodon sp.) + mistura concentrada constituída de fubá de milho (Zea mays L.), farelo de soja (Glycine max L.) e mistura mineral. As dietas foram fornecidas duas vezes ao dia, às $8 \mathrm{~h} 30$ e $16 \mathrm{~h} 30$. O comportamento ingestivo foi determinado mediante observação visual individual dos animais, durante 24 horas, a intervalos de 10 minutos para se determinar o tempo despendido em alimentação, ruminação e ócio. O tempo com alimentação e ruminação e o tempo de mastigação total aumentaram linearmente, enquanto o tempo de ócio diminuiu linearmente com o aumento do nível de FDNF nas dietas. Observaram-se efeitos linear decrescente na eficiência de alimentação e ruminação ( $\mathrm{g} \mathrm{MS} / \mathrm{h}$ ) e linear crescente no número diário de refeições, conforme amumentaram os níveis de FDNF.
\end{abstract}

Palavras-chave: alimentação, caprinos, fibra, ócio, ruminação

\section{Feeding behavior of lactating Alpine goats fed diets containing different dietary levels of forage neutral detergent fiber}

\begin{abstract}
The objective of this trial was to study the effects of different dietary levels of forage neutral detergent fiber (FNDF) on feeding behavior of lactating goats. Fifty Alpine goats were randomly assigned to five treatments (ten replicates) in a completely randomized design. Animals had free access to diets containing: 20, 27, 34, 41 or $48 \%$ of FNDF. The total mixed ration was constituted of Tifton-85 (Cynodon sp.) hay and concentrate composed of ground corn (Zea mays L.), soybean meal (Glycine max L.), and minerals. Diets were fed twice a day at 8:30 AM and 4:30 PM. Feeding behavior was determined by individual appraisal of animals every 10 minutes during 24 hours to measure eating, ruminating, and resting times. Eating, ruminating, and total chewing times increased linearly whereas the opposite was observed for resting time when FNDF levels ranged from 20 to $48 \%$ of the diet. Linear decreases on feeding and rumination efficiencies both expressed as $\mathrm{g} \mathrm{DM} / \mathrm{h}$, and a linear increase in the number of daily meals were observed with the increment of dietary FNDF.
\end{abstract}

Key Words: feed intake, goats, fiber, resting time, rumination

\section{Introdução}

Para que ocorra alta produção de leite durante a lactação, é necessária a maximização do consumo de alimento. Todavia, os animais leiteiros normalmente não consomem quantidades de energia suficientes para atender seu requerimento, pois, nesse período, a capacidade do rúmen-retículo pode limitar fisicamente o consumo.

Entretanto, quando a produção de leite aumenta, é essencial a redução no efeito de enchimento das dietas para se maximizar o consumo de energia e evitar a excessiva mobilização de reservas corporais, o que poderia provocar distúrbios metabólicos e, conseqüentemente, reduzir o desempenho animal (Dado \& Allen, 1995).

Uma vez que o conteúdo de fibra (FDN) na ração é inversamente relacionado ao de energia líquida, ou seja, maiores teores de fibra implicam menores teores de energia líquida (Mertens, 1996), não é possível o atendimento dos requerimentos nutricionais de fêmeas leiteiras de alta produção somente com volumosos, em razão da baixa densidade energética desses alimentos e de sua lenta taxa de degradação e passagem, o que limita a ingestão, como resultado do

Correspondências devem ser enviadas para: sergiocarvalho@feevale.br 
efeito de enchimento ruminal. Portanto, o aumento da utilização de concentrados é necessário por reduzir a concentração de volumosos e de fibra nas dietas.

No entanto, ruminantes requerem um mínimo de fibra efetiva na dieta para o funcionamento normal do rúmen e, particularmente, os produtores de leite necessitam de fibra para manutenção do conteúdo normal de gordura no leite. O funcionamento do rúmen está associado à adequada ruminação, que produz quantidade suficiente de substâncias tamponantes, por meio da salivação e, com isso, mantém $\mathrm{pH}$ ótimo para ação dos microrganismos celulolíticos, os quais promovem aumento na relação acetato:propionato no líquido ruminal (Santini et al., 1992).

A efetividade de uma fonte de fibra na dieta pode ser definida como a habilidade desta fonte em estimular a mastigação e/ou manter o teor normal de gordura do leite (Grant, 1997). Segundo Rodrigues (1998), o termo efetividade da fibra é confuso porque essa medida tem sido obtida utilizando-se diversas formas de resposta do animal. Para melhor entendimento, deve-se estabelecer uma distinção entre os conceitos de FDN fisicamente efetiva (FDNfe) e FDN efetiva (FDNe).

A FDNfe está relacionada às características físicas da fibra que influenciam a atividade mastigatória e a natureza bifásica do conteúdo ruminal. A FDNe, por sua vez, está relacionada à soma total da habilidade de um alimento em substituir a forragem ou o volumoso em uma ração, de modo que a porcentagem de gordura do leite produzida pelos animais que ingerem esta ração seja efetivamente mantida.

A resposta animal associada à FDNfe é a atividade de mastigação e, portanto, está diretamente relacionada à saúde animal e à redução do teor de gordura do leite, em função do pH ruminal. Segundo Lammers et al. (1996), é necessário adequado conteúdo de FDNfe para que haja bom funcionamento do rúmen. Reduções no teor de FDNfe da dieta promovem decréscimo no tempo gasto com mastigação (comendo e ruminando), acarretando declínio no $\mathrm{pH}$ ruminal, como resultado do menor fluxo de saliva para o rúmen, e diminuindo o fluxo de substâncias tamponantes, o que pode comprometer a saúde e a produtividade animal.

Para determinação da efetividade física (FDNfe) de um alimento, tem-se adotado o comportamento ingestivo dos animais, analisado em relação aos tempos com alimentação, ruminação e ócio e às eficiências de alimentação e ruminação.

De acordo com Albright (1993), os períodos utilizados para alimentação, ruminação e ócio geralmente variam consideravelmente de acordo com o manejo e o tipo de dieta fornecida.

Van Soest (1994) afirma que o tempo de ruminação é diretamente proporcional ao conteúdo de FDN e à forma física da dieta. Dado \& Allen (1995), no entanto, enfatizam que o número de períodos em que se observa ruminação eleva com o aumento do conteúdo de fibra, refletindo a necessidade de processar a digesta ruminal, maximizando a eficiência digestiva.

Essas informações corroboram os valores observados por Beauchemin (1991), que verificou aumento no tempo de ruminação de 402 para 443 minutos/dia quando o teor de FDN da dieta elevou de 31 para $37 \%$. Comportamento semelhante foi verificado por Pereira et al. (1998), que observaram aumento de $20,7 \%$ no tempo diário de ruminação quando a FDN da dieta passou de 40,85 para $59,82 \%$.

A eficiência de ruminação é importante também para o controle da utilização de alimentos de baixa digestibilidade, pois o animal pode ruminar maior quantidade de alimentos de baixa qualidade por tempo de ruminação, proporcionando maior consumo de alimentos e melhor desempenho produtivo (Welch, 1982). Em dietas com alto conteúdo de FDN, a eficiência de ruminação e mastigação é diminuída, em razão da maior dificuldade em reduzir o tamanho das partículas originadas de materiais ricos em fibra, o que diminui a ingestão de alimento (Dulphy et al., 1980).

Este trabalho foi conduzido com o objetivo de avaliar o comportamento ingestivo de cabras Alpinas em lactação alimentadas com dietas contendo diferentes níveis de fibra em detergente neutro proveniente da forragem para melhor compreensão dos processos que controlam a ingestão de alimento e, conseqüentemente, o desempenho animal.

\section{Material e Métodos}

O experimento foi realizado no Setor de Caprinocultura do Departamento de Zootecnia, do Centro de Ciências Agrárias da Universidade Federal de Viçosa.

O município de Viçosa está localizado na Zona da Mata, Minas Gerais, a $657 \mathrm{~m}$ de altitude, 20 $25^{\circ} 20^{\prime \prime}$ de latitude sul e 42052'40" de longitude oeste. Apresentou em 1999 precipitação pluviométrica de $1.224,8 \mathrm{~mm}$, umidade relativa média do ar de $78,42 \%$, temperatura média de $18,61^{\circ} \mathrm{C}$, médias das máximas e mínimas de 26,83 e $14,83^{\circ} \mathrm{C}$ e insolação média de 6,01 horas (UFV, 2001). O clima da região é do tipo Cwa, segundo classificação de Köeppen (1948).

Utilizaram-se 50 cabras Alpinas em lactação, com peso vivo médio de $57,73 \mathrm{~kg}$, introduzidas no experimento no 45 응 dia pós-parto e mantidas até o 105‥ Os 45 dias iniciais da lactação foram destinados à adaptação dos animais às dietas, às instalações e ao manejo.

Os animais foram alimentados duas vezes ao dia, às $8 \mathrm{~h} 30 \mathrm{e} 16 \mathrm{~h} 30$, em baias individuais $(1,5 \times 2,0 \mathrm{~m})$ providas de comedouro e bebedouro, com piso totalmente ripado. 
Foram testadas cinco dietas constituídas de diferentes níveis de FDN proveniente da forragem (FDNF), corrigida para cinzas. Os níveis de FDNF foram 20, 27, 34, 41 e $48 \%$, com base na matéria seca (MS). Foi utilizada uma ração completa de feno de tifton-85 (Cynodon sp.) + mistura concentrada. A relação volumoso:concentrado variou conforme o tratamento, de modo a se atingir o nível de FDNF pretendido para as dietas experimentais, constituídas de fubá de milho (Zea mays L.), farelo de soja (Glycine $\max$ L.) e mistura mineral. As dietas utilizadas foram formuladas para ser isoprotéicas e conter $18 \%$ de PB (MS).

A composição percentual dos ingredientes consta na Tabela 1 e a composição bromatológica das dietas experimentais, na Tabela 2 .

$\mathrm{O}$ feno de tifton 85 foi picado em moinho de martelo, obtendo-se partículas de 1 a $3 \mathrm{~cm}$, e armazenado em sacos. $\mathrm{O}$ alimento fornecido e as sobras foram pesados diariamente para o controle do consumo diário de matéria seca pelos animais. A quantidade a ser oferecida foi ajustada de acordo com a sobra diária, que deveria ser de $10 \%$ da quantidade oferecida no dia anterior, para garantir consumo voluntário máximo pelos animais.

Coletaram-se diariamente, individualmente, amostras das sobras e dos alimentos, que formaram amostras, destinadas às análises laboratoriais. Os teores de MS, MO, PB, EE, FDN, FDA, LIG (método do permanganato de potássio) e CIN foram determinados conforme descrito por Silva (1990). Os carboidratos totais (CHT) foram estimados pela diferença de $100-(\% \mathrm{~PB}+\% \mathrm{EE}+\% \mathrm{CIN})$, de acordo com procedimentos

Tabela 1 - Composição percentual dos ingredientes das dietas experimentais (\%MS)

Table 1 - Ingredient composition of the experimental diets on dry matter basis

\begin{tabular}{|c|c|c|c|c|c|}
\hline \multirow[t]{2}{*}{$\begin{array}{l}\text { Ingrediente ( } \% \text { MS }) \\
\text { Ingredient }(\% D M)\end{array}$} & \multicolumn{5}{|c|}{$\begin{array}{l}\text { Nível de FDNF } \\
\text { Level of FNDF }\end{array}$} \\
\hline & 20 & 27 & 34 & 41 & 48 \\
\hline $\begin{array}{l}\text { Feno de tifton } 85 \\
\text { Tifton } 85 \text { hay }\end{array}$ & 28,81 & 38,89 & 48,97 & 59,06 & 69,14 \\
\hline $\begin{array}{l}\text { Fubá de milho } \\
\text { Ground corn }\end{array}$ & 46,85 & 39,17 & 31,46 & 23,71 & 16,00 \\
\hline $\begin{array}{l}\text { Farelo de soja } \\
\text { Soybean meal }\end{array}$ & 21,84 & 19,54 & 17,25 & 14,96 & 12,67 \\
\hline $\begin{array}{l}\text { Calcário calcítico } \\
\text { Limestone }\end{array}$ & 1,74 & 1,64 & 1,56 & 1,36 & 1,18 \\
\hline $\begin{array}{l}\text { Fosfato bicálcico } \\
\text { Dicalcium phosfate }\end{array}$ & 0 & 0 & 0 & 0,15 & 0,25 \\
\hline $\begin{array}{l}\text { Sal comum } \\
\text { Salt }\end{array}$ & 0,74 & 0,74 & 0,74 & 0,74 & 0,74 \\
\hline Premix $^{1}$ & 0,02 & 0,02 & 0,02 & 0,02 & 0,02 \\
\hline
\end{tabular}

1 Composição (Composition): sulfato de zinco (zinc sulfate), $50 \%$; sulfato de cobre (copper sulfate), $41,6 \%$; sulfato de cobalto (cobalt sulfate), $2,8 \%$; selenito de sódio (sodium selenite), $2,8 \%$; iodato de potássio (potassium iodate), $2,8 \%$.
Tabela 2 - Composição bromatológica das dietas experimentais Table 2 - Chemical composition of experimental diets

\begin{tabular}{|c|c|c|c|c|c|}
\hline \multirow[t]{2}{*}{ Item $(\%)$} & \multicolumn{5}{|c|}{$\begin{array}{l}\text { Nível de FDNF } \\
\text { Level of FNDF }\end{array}$} \\
\hline & 20 & 27 & 34 & 41 & 48 \\
\hline $\begin{array}{l}\text { Matéria seca } \\
\text { Dry matter }\end{array}$ & 84,65 & 84,27 & 83,90 & 83,53 & 83,15 \\
\hline $\begin{array}{l}\text { Matéria orgânica } \\
\text { Organic matter }\end{array}$ & 93,78 & 93,50 & 93,19 & 92,86 & 92,56 \\
\hline $\begin{array}{l}\text { Proteína bruta } \\
\text { Crude protein }\end{array}$ & 18,41 & 18,37 & 18,33 & 18,29 & 18,25 \\
\hline $\begin{array}{l}\text { Extrato etéreo } \\
\text { Ether extract }\end{array}$ & 2,35 & 2,23 & 2,10 & 1,98 & 1,86 \\
\hline $\begin{array}{l}\text { FDN total } \\
\text { Total NDF }\end{array}$ & 30,18 & 36,60 & 43,01 & 49,43 & 55,85 \\
\hline $\begin{array}{l}\text { FDNF } \\
F N D F\end{array}$ & 20,00 & 27,00 & 34,00 & 41,00 & 48,00 \\
\hline $\begin{array}{l}\mathrm{FDA} \\
A D F\end{array}$ & 17,97 & 21,55 & 25,13 & 28,71 & 32,28 \\
\hline $\begin{array}{l}\mathrm{CHT} \\
\text { Total carbohydrate }\end{array}$ & 73,02 & 72,9 & 72,76 & 72,59 & 72,45 \\
\hline $\begin{array}{l}\mathrm{CNE} \\
N S C\end{array}$ & 46,57 & 40,41 & 34,24 & 28,03 & 21,85 \\
\hline $\begin{array}{l}\text { CNE:FDN total } \\
\text { Total NSC:NDF }\end{array}$ & $1,54: 1$ & $1,10: 1$ & $0,80: 1$ & $0,57: 1$ & $0,39: 1$ \\
\hline $\begin{array}{l}\text { Lignina } \\
\text { Lignin }\end{array}$ & 1,32 & 1,65 & 1,99 & 2,33 & 2,66 \\
\hline $\begin{array}{l}\text { Cinzas } \\
\text { Ash }\end{array}$ & 6,22 & 6,50 & 6,81 & 7,14 & 7,44 \\
\hline $\begin{array}{l}\text { Energia líquida }{ }^{1} \\
\text { Net energy }\end{array}$ & 1,59 & 1,49 & 1,38 & 1,27 & 1,16 \\
\hline $\begin{array}{l}\text { Cálcio } \\
\text { Calcium }\end{array}$ & 0,80 & 0,80 & 0,80 & 0,80 & 0,80 \\
\hline $\begin{array}{l}\text { Fósforo } \\
\text { Phosphorus }\end{array}$ & 0,3 & 0,3 & 0,3 & 0,3 & 0,3 \\
\hline
\end{tabular}

1 Valor calculado (Calculated value).

adotados por Sniffen et al. (1992), e os carboidratos nãoestruturais (CNE), pela diferença CHT - FDNc.

O conteúdo de energia líquida (EL) do volumoso foi calculado utilizando-se a equação de Moe \& Tyrrel (1976): $\mathrm{EL}=0,0245 \times$ \%NDT $-0,12$. O NDT foi estimado a partir da equação descrita por Van Soest (1994), em que: NDT = DMS - cinzas total $+1,25$ extrato etéreo + 1,9, em que DMS $($ digestibilidade da matéria seca $)=((100-\operatorname{FDNc} x(0,98))$ $+($ FDN total $x$ digestibilidade da FDN corrigida para Kd e $\mathrm{Kp})$ - 12,9. A digestibilidade da FDN corrigida para $\mathrm{Kd} \mathrm{e}$ $\mathrm{Kp}$ foi obtida considerando-se $\mathrm{Kp}$ de $0,05 \mathrm{~h}^{-1}$ e $\mathrm{Kd}$ de $0,0256 \mathrm{~h}^{-1}$ e o valor de $\mathrm{Kd}$, aplicando-se análise de regressão linear (Waldo, 1970) aos dados de digestibilidade da FDN, segundo Van Soest (1994), com tempo de digestão de 96 horas.

O comportamento ingestivo foi determinado mediante observação visual individual dos animais, durante 24 horas, a intervalos de 10 minutos para determinação do tempo despendido em alimentação, ruminação e ócio. Na observação noturna dos animais, o ambiente foi mantido com iluminação artificial. 
Os resultados referentes aos fatores do comportamento ingestivo foram obtidos utilizando-se as equações:

$$
\begin{aligned}
\mathrm{EAL}_{\mathrm{MS}} & =\mathrm{CMS} / \mathrm{TAL} ; \\
\mathrm{EAL}_{\mathrm{FDN}} & =\mathrm{CFDN} / \mathrm{TAL} ;
\end{aligned}
$$

em que: $\operatorname{EAL}_{\mathrm{MS}}$ (g MS consumida/h); $\mathrm{EAL}_{\mathrm{FDN}}(\mathrm{g}$ FDN consumida/h) = eficiência de alimentação; $\mathrm{CMS}(\mathrm{g})=$ consumo diário de matéria seca; $\operatorname{CFDN}(\mathrm{g})=$ consumo diário de FDN; $\mathrm{TAL}=$ tempo gasto diariamente em alimentação.

$$
\begin{aligned}
\mathrm{ERU}_{\mathrm{MS}} & =\mathrm{CMS} / \mathrm{TRU} ; \\
\mathrm{ERU}_{\mathrm{FDN}} & =\mathrm{CFDN} / \mathrm{TRU} ;
\end{aligned}
$$

em que: $\operatorname{ERU}_{\mathrm{MS}}$ (g MS ruminada/h); $\mathrm{ERU}_{\mathrm{FDN}}(\mathrm{g}$ FDN ruminada/h $)=$ eficiência de ruminação e TRU $(\mathrm{h} /$ dia $)=$ tempo de ruminação.

$$
\mathrm{TMT}=\mathrm{TAL}+\mathrm{TRU}
$$

em que: TMT $(\mathrm{min} /$ dia $)=$ tempo de mastigação total.

O delineamento experimental utilizado foi o inteiramente casualizado com cinco tratamentos e dez repetições, em que a cabra constituiu a unidade experimental. A análise estatística foi realizada de acordo com os procedimentos descritos por Searle (1971), utilizando-se a produção de leite inicial como covariável. Quando foi verificado efeito significativo da covariável $(\mathrm{P} \leq 0,05)$ pelo teste $\mathrm{F}$, realizaram-se análises de variância e de regressão.

Quando não houve efeito significativo da covariável ( $\mathrm{P}>0,05)$, os dados foram interpretados por meio de análises de variância e regressão, utilizando-se o programa SAEG Sistema de Análises Estatísticas e Genéticas (UFV, 1995).

Os modelos foram selecionados com base no coeficiente de determinação e na significância dos coeficientes de regressão, a 5\% de probabilidade, utilizando-se o teste F.

\section{Resultados e Discussão}

Os resultados referentes aos tempos despendidos em alimentação, ruminação ou ócio, o tempo de mastigação total e os tempos em que os animais permaneceram em pé ou deitados, em função do nível de FDNF das dietas, encontram-se na Tabela 3.

A elevação do nível de FDNF das dietas experimentais proporcionou aumento linear $(\mathrm{P}<0,01)$ nos tempos despendidos em alimentação e ruminação e redução do tempo de ócio. Os tempos de permanência em pé ou deitados não foram influenciados, apresentado valores médios de 593,67 e 846,33 min/dia, respectivamente. O tempo de mastigação total aumentou linearmente $(\mathrm{P}<0,01)$ com a elevação do teor de fibra nas dietas, como conseqüência dos aumentos verificados nos tempos gastos com alimentação e ruminação.

O aumento do tempo em alimentação, com a elevação do teor de FDNF, pode ser conseqüência da proporção de forragem na dieta. Uma vez que o conteúdo de fibra está inversamente relacionado ao conteúdo de energia líquida das dietas (Mertens, 1996), o incremento verificado no período de alimentação, em minutos/dia, pode ser explicado, em parte, pela diminuição da densidade energética das dietas, ocasionada pela elevação do teor de FDNF. Possivelmente, a maior procura por alimento durante o dia tenha decorrido da necessidade dos animais de aumentar o consumo para atender o requerimento de energia, uma vez que a concentração desse nutriente era menor nas rações com maior conteúdo de FDNF.

O aumento linear no tempo gasto com ruminação com o aumento do nível de FDNF das dietas está em acordo com os resultados descritos por Van Soest (1994), que afirma que o tempo gasto em ruminação é proporcional ao teor de parede celular dos alimentos. Confirma também os dados reportados por Church (1988), segundo o qual forragens com alto conteúdo de FDN necessitam de maior período de ruminação, refletindo em maior necessidade de processar a fibra da dieta para passar através do trato digestivo do ruminante.

Os valores observados para os tempos em alimentação e ruminação corroboram aqueles verificados por Santini et al. (1992), que trabalharam com cabras Alpinas em lactação e constataram aumento linear de 180 para $263 \mathrm{~min} /$ dia e de 249 para $364 \mathrm{~min} /$ dia nos tempos de alimentação e de ruminação, quando o teor de FDN das dietas elevou de 38,3 para $47,4 \%$. Confirman também os descritos por Rabelo (2002), que, utilizando dietas contendo diferentes níveis de FDN $(37,7 ; 41,6 ; 44,0$ e 45,4\%) na alimentação de bovinos de corte, verificou tendência de aumento do tempo em alimentação e redução no tempo em ócio $(\mathrm{P}=0,08)$, conforme elevou o teor de FDN nas dietas.

Os valores médios observados para os tempos de alimentação (295,51 min/dia) e de ruminação (394,42 min/dia) foram próximos aos citados por Church (1988) para cabras, de $254 \mathrm{e}$ $446 \mathrm{~min} /$ dia para alimentação e ruminação, respectivamente, e aos observados por Santini et al. (1992), que, trabalhando com cabras Alpinas em lactação, verificaram tempo máximo de alimentação de $263 \mathrm{~min} /$ dia e de ruminação de $364 \mathrm{~min} / \mathrm{dia}$, ao utilizarem uma dieta contendo $47,4 \%$ de FDN.

A distribuição da porcentagem de alimentação e ruminação, em quatro períodos, nas 24 horas do dia, conforme 
os níveis de FDNF das dietas experimentais, é apresentada nas Figuras 1 e 2.

A soma dos períodos 2 e 3 representou, em média, $70,2 \%$ do período total de alimentação (Figura 1), o que pode ser, em parte, explicado pelo manejo alimentar adotado, possibilitando concentração da atividade de ingestão em torno dos dois horários de distribuição da ração (8h30 e 16h30). Essa observação confirma o estímulo da distribuição de ração sobre a atividade de alimentação (Fischer et al., 1998) e a concentração da atividade ingestiva durante o período diurno (Miranda et al., 1999). Pode-se verificar, também, que o período entre 18 e $24 \mathrm{~h}$ representou, em média, $18,52 \%$ da ingestão total de alimento, como conseqüência do horário de alimentação da tarde, que foi próximo ao início do quarto período.

A maior concentração da atividade de ruminação ocorreu no horário noturno (Figura 2) e o período 1 correspondeu a $38,78 \%$ do tempo total de ruminação. A atividade de ruminação diurna ocorreu principalmente no início do período 2 , antes do horário do fornecimento da ração, ou seja, das $6 \mathrm{~h}$ às $8 \mathrm{~h} 30$, e correspondeu a $28,17 \%$ da ruminação total. A afirmativa de Deswysen et al. (1993) de que os animais, quando alimentados duas vezes ao dia, às $9 \mathrm{~h}$ e entre 16 e $18 \mathrm{~h}$, apresentam importante período de ruminação no início da manhã, em adição ao pico noturno, corrobora os resultados apresentados neste estudo. Os resultados obtidos também confirmam os achados de Fischer et al. (1998), que, em ovinos, verificaram que a atividade de ruminação foi mais intensa durante a noite, nas primeiras horas do dia e de 11 às $15 \mathrm{~h}$.

Os resultados concernentes aos consumos de MS e FDN, em kg/dia e \%PV, às eficiências de alimentação (EAL) e ruminação (ERU), expressas em g MS/h e g FDN/h, de acordo com os níveis de FDNF das dietas experimentais, encontram-se na Tabela 4.

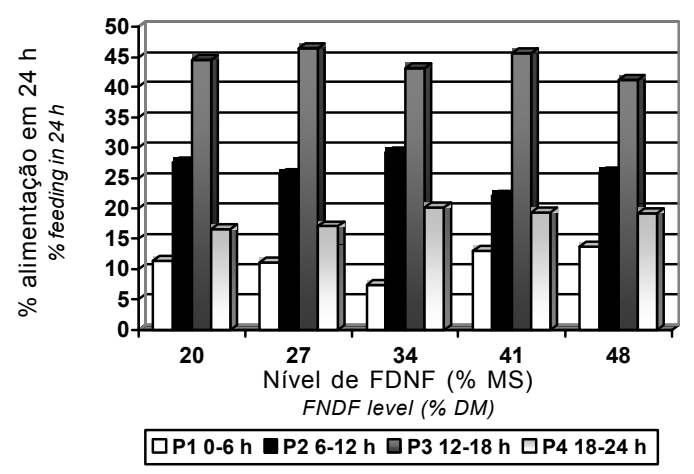

Figura 1 - Porcentagem de alimentação, em quatro períodos, nas 24 horas do dia.

Figure 1 - Percentage of feeding, in four periods, in a 24 hours feeding cycle.

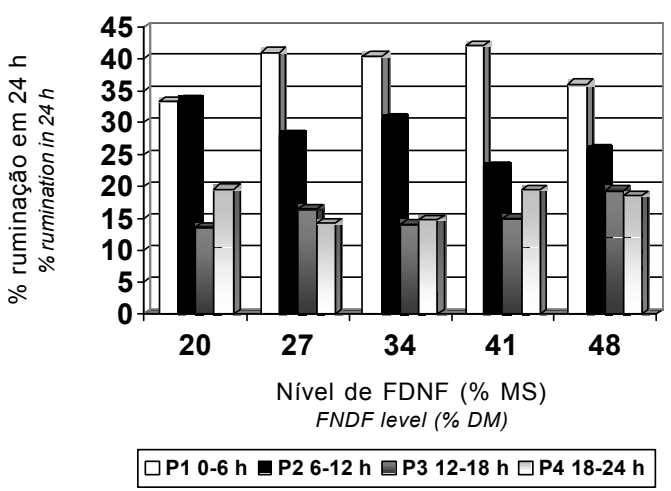

Figura 2 - Porcentagem de ruminação, em quatro períodos, nas 24 horas do dia.

Figure 2 - Percentage of rumination, in four periods, in a 24 hours feeding cycle.

Os consumos de MS dos animais, em $\mathrm{kg} / \mathrm{dia}$ e \% PV, diminuíram linearmente com o incremento dos níveis de FDNF da ração, comportamento inverso ao verificado para o consumo de FDN que aumentou linearmente.

Tabela 3 - Médias do tempo despendido em alimentação (Alim.), ruminação (Rum.) e ócio (Ócio), do tempo de mastigação total (TMT), tempo de permanência em pé (Em pé) ou deitada (Deit.), em minutos por dia (min/dia), conforme os níveis de FDNF das dietas experimentais

Table 3 - $\quad$ Average time spent eating (Eat.), ruminating (Rum.), and idling (IDL.), total chewing time (TCT), time standing (STD.) or lying (LYI.), according to the different dietary FNDF levels

\begin{tabular}{|c|c|c|c|c|c|c|c|}
\hline \multirow[b]{2}{*}{ Item } & \multicolumn{5}{|c|}{$\begin{array}{c}\text { Nível de FDNF (\%) } \\
\text { Level of FNDF }\end{array}$} & \multirow[b]{2}{*}{ ER } & \multirow[b]{2}{*}{ CV (\%) } \\
\hline & 20 & 27 & 34 & 41 & 48 & & \\
\hline Alim. (Eat.) & 235,72 & 271,65 & 294,40 & 326,64 & 349,15 & 1 & 24,36 \\
\hline Rum. (Rum.) & 311,14 & 360,06 & 393,08 & 437,55 & 470,28 & 2 & 19,92 \\
\hline $\mathrm{TMT}(T C T)$ & 546,86 & 631,72 & 687,48 & 764,19 & 819,43 & 3 & 18,66 \\
\hline Ócio (IDL.) & 893,14 & 808,28 & 752,51 & 675,81 & 620,56 & 4 & 17,31 \\
\hline Em pé (STD.) & 593,33 & 599,00 & 562,00 & 581,00 & 633,00 & $Y=593,67$ & 15,94 \\
\hline Deit. (LYI.) & 846,67 & 841,00 & 878,00 & 859,00 & 807,00 & $Y=846,33$ & 11,18 \\
\hline
\end{tabular}

1. $\hat{\mathrm{Y}}=70,4852+4,0810 * * \mathrm{FDNF}, \mathrm{R}^{2}=0,24 ; 2 . \hat{\mathrm{Y}}=95,8355+5,7197 * * \mathrm{FDNF}, \mathrm{R}^{2}=0,36 ; 3 . \hat{\mathrm{Y}}=166,3207+9,8008 * * \mathrm{FDNF}, \mathrm{R}^{2}=0,38$

4. $\hat{\mathrm{Y}}=1273,679-9,8008 * * \mathrm{FDNF}, \mathrm{R}^{2}=0,38$.

* Significativo a $1 \%$ de probabilidade pelo teste F; FDNF = nível de FDN proveniente da forragem nas dietas.

** Significant at $1 \%$ of probability by $F$ test; $F N D F=$ Level of forage neutral detergent fiber in the diets. 
A eficiência de alimentação, quando expressa em $\mathrm{g}$ $\mathrm{MS} / \mathrm{h}$, reduziu de forma linear $(\mathrm{P}<0,01)$, mas, quando expressa em $\mathrm{g} \mathrm{FDN} / \mathrm{h}$, apesar de não ter sido observado efeito significativo, apresentou comportamento crescente com o aumento do nível de FDNF. O valor médio verificado para a eficiência de alimentação foi de $185,8 \mathrm{~g} \mathrm{FDN} / \mathrm{h}$. A menor ingestão de MS e maior de FDN observadas com o incremento dos níveis de FDN da dieta podem ser consideradas a principal causa da pior e melhor eficiência, respectivamente. Trabalhando com bezerros holandeses alimentados com dietas contendo níveis de concentrado de 30 a $90 \%$ da MS, o que levou a níveis de FDN de 62,43 a 20,66\%, Bürger et al. (2000) encontaram efeitos linear decrescente para a eficiência de alimentação, quando expressa em $\mathrm{g} \mathrm{MS} / \mathrm{h}$, e crescente, quando expressa em $\mathrm{g}$ FDN/h, com a redução do nível de concentrado e o aumento do nível de FDN na ração, o que concorda com os resultados verificados neste experimento.

Observou-se efeito linear decrescente na eficiência de ruminação, em g MS/h, com o aumento do nível de FDNF e, portanto, a melhor eficiência foi obtida com o nível de $20 \%$ de FDNF. A eficiência de ruminação, quando relacionada à FDN, não diferiu entre os tratamentos, apresentando valor médio de 138,2 g FDN/h. A eficiência de ruminação melhorou com a redução do nível de FDNF, em razão da maior ingestão de MS e do menor tempo de ruminação ( $\min / \mathrm{kg} \mathrm{MS}$ ), o que resultou em valores maiores.

Constam na Tabela 5 as médias do número de refeições diárias e o tempo despendido por refeição, em minutos. $\mathrm{O}$ aumento linear crescente $(\mathrm{P}<0,05)$ no número de refeições pode ser atribuído à menor densidade energética da dieta com o aumento do teor de fibra, que induziu os animais

Tabela 4 - Médias dos consumos de MS (CMS) e FDN (CFDN), eficiência de alimentação (EAL) e eficiência de ruminação (ERU), em função dos níveis de FDNF das dietas experimentas

Table 4 - Means of intakes of DM (DMI) and NDF (NDFI), feed efficiency (FEF) and rumination efficiency (RUE), according to the different dietary FNDF levels

\begin{tabular}{|c|c|c|c|c|c|c|c|}
\hline \multirow[b]{2}{*}{ Item } & \multicolumn{5}{|c|}{$\begin{array}{c}\text { Nível de FDNF (\%) } \\
\text { Level of FNDF }\end{array}$} & \multirow[b]{2}{*}{ ER } & \multirow[b]{2}{*}{ CV (\%) } \\
\hline & 20 & 27 & 34 & 41 & 48 & & \\
\hline $\begin{array}{l}\text { CMS (kg/dia) } \\
\text { DMI (kg/day) }\end{array}$ & 2,784 & 2,777 & 2,605 & 2,558 & 2,383 & 1 & 10,82 \\
\hline $\begin{array}{l}\text { CMS (\% PV) } \\
D M I(\% B W)\end{array}$ & 4,86 & 4,85 & 4,57 & 4,49 & 4,20 & 2 & 14,09 \\
\hline $\begin{array}{l}\text { CFDN (kg/dia) } \\
\text { NDFI (kg/day) }\end{array}$ & 0,624 & 0,774 & 0,871 & 1,006 & 1,102 & 3 & 12,38 \\
\hline $\begin{array}{l}\text { CFDN (\% PV) } \\
\text { NDFI }(\% B W)\end{array}$ & 1,09 & 1,36 & 1,53 & 1,77 & 1,94 & 4 & 14,00 \\
\hline $\begin{array}{l}\text { EAL }(\mathrm{g} \mathrm{MS} / \mathrm{h}) \\
F E F(g D M / h)\end{array}$ & 668,27 & 676,02 & 571,29 & 497,37 & 433,81 & 5 & 26,67 \\
\hline $\begin{array}{l}\text { EAL }(\mathrm{g} \text { FDN/h) } \\
F E F(g N F / h)\end{array}$ & 152,20 & 185,21 & 193,23 & 195,84 & 199,52 & $\hat{\mathrm{Y}}=185,8$ & 27,97 \\
\hline $\begin{array}{l}\text { ERU }(\mathrm{g} \mathrm{MS} / \mathrm{h}) \\
R U E(g D M / h)\end{array}$ & 595,73 & 524,75 & 370,31 & 356,38 & 316,93 & 6 & 32,47 \\
\hline $\begin{array}{l}\text { ERU }(\mathrm{g} \mathrm{FDN} / \mathrm{h}) \\
R U E(g N D F / h)\end{array}$ & 135,46 & 143,72 & 125,30 & 140,19 & 146,19 & $\hat{\mathrm{Y}}=138,2$ & 26,90 \\
\hline
\end{tabular}

1. $\hat{Y}=2,1463-0,014{ }^{* *} F D N F, R^{2}=0,20 ; 2 . \hat{Y}=3,6059-0,0229{ }^{*} F D N F, R^{2}=0,12 ; 3 . \hat{Y}=-0,0583+0,0179{ }^{* *} F D N F, R^{2}=0,73 ; 4 . \hat{Y}=-0,1290+0,0302$ ${ }^{* *} \mathrm{FDNF}, \mathrm{R}^{2}=0,68 ; 5 . \hat{\mathrm{Y}}=887,696-9,3439{ }^{* *} \mathrm{FDNF}, \mathrm{R}^{2}=0,94 ; 6 . \hat{\mathrm{Y}}=783,221-10,3170{ }^{* *} \mathrm{FDNF}, \mathrm{R}^{2}=0,90$

${ }^{*} e^{* *}$ Significativo a 5 e $1 \%$ de probabilidade, respectivamente, pelo teste F; FDNF = nível de FDN proveniente da forragem nas dietas. ${ }^{*}$ and ${ }^{* *}$ Significant at 5 and $1 \%$ probability, respectively, by $F$ test; FNDF = dietary forage FNDFlevel.

Tabela 5 - Médias do número de refeições diárias e do tempo despendido por refeição, em função dos níveis de FDNF das dietas experimentais

Table 5 - Means of number of daily meals and of the time spent per meal according to the different dietary FNDF levels

\begin{tabular}{|c|c|c|c|c|c|c|c|}
\hline \multirow[b]{2}{*}{ Item } & \multicolumn{5}{|c|}{$\begin{array}{c}\text { Nível de FDNF (\%) } \\
\text { Level of FNDF }\end{array}$} & \multirow[b]{2}{*}{ ER } & \multirow[b]{2}{*}{ CV (\%) } \\
\hline & 20 & 27 & 34 & 41 & 48 & & \\
\hline $\begin{array}{l}\text { Número de refeições } \\
\text { Number of meals }\end{array}$ & 11,56 & 11,80 & 11,90 & 13,10 & 14,20 & 1 & 21,96 \\
\hline $\begin{array}{l}\text { Tempo/refeição (min) } \\
\text { Time/meal (min) }\end{array}$ & 22,94 & 22,98 & 23,13 & 24,58 & 26,37 & $\bar{Y}=24,02$ & 24,60 \\
\hline
\end{tabular}

1. $\mathrm{Y}=9,2611+0,1115 * \mathrm{FDNF}, \mathrm{R}^{2}=0,87$

* Significativo a $5 \%$ de probabilidade pelo teste F; FDNF = nível de FDN proveniente da forragem nas dietas.

* Significant at $5 \%$ of probability by F test; FNDF = dietary forage FNDF level. 
a procurar mais vezes durante o dia o alimento no comedouro, como forma de minimizar o déficit desse nutriente na ração.

O tempo médio por refeição não foi influenciado pelo nível de FDNF da dieta e a duração de cada refeição foi, em média, de 24,02 minutos. Miranda et al. (1999), trabalhando com novilhas mestiças Holandês-Zebu alimentadas com uma dieta contendo em torno de 59\% de FDN, encontrou média de 11,62 refeições/dia, com duração média de 28,70 minutos, próxima à verificada neste estudo.

\section{Conclusões}

O aumento da quantidade de fibra na ração de cabras em lactação acarretou incremento do tempo gasto diariamente em alimentação e ruminação e, portanto, promoveu aumento no tempo diário despendido em mastigação total. Como conseqüência, houve diminuição do tempo de ócio.

Verificou-se diminuição na eficiência de alimentação e ruminação com o incremento do teor de FDNF das rações, o que pode levar à limitação da ingestão diária de alimento;

O número de refeições diárias aumentou com o incremento do teor de fibra das rações, provavelmente, em razão da diminuição da densidade energética, com o aumento do teor de FDNF das rações.

\section{Literatura Citada}

ALBRIGHT, J.L. Feeding behavior of dairy cattle. Journal of Dairy Science, v.76, n.2, p.485-498, 1993.

BEAUCHEMIN, K.A. Effects of dietary neutral fiber concentration and alfafa hay quality on chewing, rumen function, and milk production of dairy cows. Journal of Dairy Science, v.74, n.9, p.3140-3151, 1991.

BURGER, P.J.; PEREIRA, J.C.; QUEIROZ, A.C. et al. Comportamento ingestivo em bezerros Holandeses alimentados com dietas contendo diferentes níveis de concentrado. Revista Brasileira de Zootecnia, v.29, n.1, p.236-242, 2000.

CHURCH, D.C. El ruminat: fisiología digestiva y nutrición. Zaragoza: Acribia, 1988. 641p.

DADO, R.G.; ALLEN, M.S. Intake limitation, feeding behavior, and rumen function of cows challenged with rumen fill from dietary fiber or inert bulk. Journal of Dairy Science, v.78, n.1, p.118-133, 1995.

DESWYSEN, A.G.; DUTILlEUL, P.; GODFRIN, J.P. et al. Nycterohemeral eating and ruminating pattern in heifers fed grass or corn silage: analisis by finite fourier transform. Journal of Animal Science, v.71, n.10, p.2739-2747, 1993.

DULPHY, J.P.; REMOND, B.; THERIEZ, M. Ingestive behavior and related activities in ruminants. In: RUCKEBUSH, Y.; THIVEND, P. (Eds). Digestive physiology and metabolism in ruminants. Lancaster: MTP, 1980. p.103-122.

FISCHER, V.; DESWYSEN, A.G.; DĖSPRES, L. et al. Padrões nictemerais do comportamento ingestivo de ovinos. Revista Brasileira de Zootecnia, v.27, n.2, p.362-369, 1998.

GRANT, R.J. Interactions among forages and nonforage fiber source. Journal of Dairy Science, v.80, p.1438-1446, 1997.
KÖEPPEN, W. Climatologia. Buenos Aires: Panamericana, 1948. $478 \mathrm{p}$.

LAMMERS, B.P.; BUCKMASTER, D.R.; HEINRICHS, A.J. A simple method for the analysis of particle sizes of forage and total mixed rations. Journal of Dairy Science, v.79, n.5, p.922928, 1996.

MERTENS, D.R. Using fiber and carbohydrate analyses to formulate dairy rations. Journal of Animal Science, v.80, n.7, p.1463$1481,1996$.

MIRANDA, L.F.; QUEIROZ, A.C.; VALADARES FILHO, S.C. et al. Comportamento ingestivo de novilhas leiteiras alimentadas com dietas à base de cana-de-açúcar. Revista Brasileira de Zootecnia, v.28, n.3, p.614-620, 1999.

MOE, P.W.; TYRREL, H.F. Estimating metabolizable and net energy of feeds. In: INTERNATIONAL SYMPOSIUM ON FEED COMPOSITION, ANIMAL NUTRIENT REQUIREMENTS, AND COMPUTERIZATION OF DIETS, 1., 1976, Logan. Proceedings... Logan: Utah State University, 1976. p.232-237.

PEREIRA, E.S.; QUEIROZ, A.C.; NEVES, J.S. et al. Níveis de fibra em dietas de novilhas leiteiras. 2. Comportamento ingestivo. In: REUNIÃO ANUAL DA SOCIEDADE BRASILEIRA DE ZoOTECNIA, 35., Botucatu. Anais... Botucatu: Sociedade Brasileira de Zootecnia, 1998. p.524-526.

RABELO, M.M.A. Efeito de fontes e níveis de fibra íntegra, em dietas contendo bagaço de cana-de-açúcar tratado sob pressão e vapor, sobre a digestibilidade, desempenho e comportamento ingestivo de bovinos de corte. Piracicaba: Escola Superior de Agricultura Luiz de Queiroz, 2002.61p. Dissertação (Mestrado em Agronomia) - Escola Superior de Agricultura Luiz de Queiroz, 2002.

RODRIGUES, M.T. Uso de fibras em rações de ruminantes. In: CONGRESSO NACIONAL DOS ESTUDANTES DE ZOOTECNIA, 1998, Viçosa, MG. Anais... Viçosa, MG: Universidade Federal de Viçosa, 1998. p.139-171.

SEARLE, S.R. Linears models. New York: John Wiley \& Sons, 1971. 533p.

SILVA, D.J. Análise de alimentos (métodos químicos e biológicos). Viçosa, MG: Universidade Federal de Viçosa, 1990. 165p.

SANTINI, F.J.; LU, C.D.; POTCHOIBA, M.J. et al. Dietary fiber and milk yield, mastication, digestion, and rate of passage in goats fed alfafa hay. Journal of Dairy Science, v.75, p.209219,1992 .

SNIFFEN, C.J.; O'CONNOR, J.D.; Van SOEST, P.J. A net carbohydrate and protein system for evaluating cattle diets: II. Carbohydrate and protein availability. Journal of Animal Science, v.70, p.3562-3577, 1992.

UNIVERSIDADE FEDERAL DE VIÇOSA - UFV. Dados climáticos. Departamento de Engenharia Agrícola. Estação Metereológica. Viçosa, MG: Universidade Federal de Viçosa, 2001. n.p.

UNIVERSIDADE FEDERAL DE VIÇOSA. SAEG - Sistema de análises estatísticas e genéticas. Viçosa, MG: 1995. (Apostila).

Van SOEST, P.J. Nutritional ecology of the ruminant. 2.ed. Ithaca: Comstock, 1994. 476p.

WALDO, D.R. Factors influencing voluntary intake of forages. In: NATIONAL CONFERENCE ON FORAGE QUALITY EVALUATION AND UTILIZATION, 1970, Lincoln. Proceedings... Lincoln: Nebraska Center for Continuing Education, 1970. p.1-22.

WELCH, J.G. Rumination, particle size and passage from the rumen. Journal of Animal Science, v.54, n.4, p.885-895, 1982. 